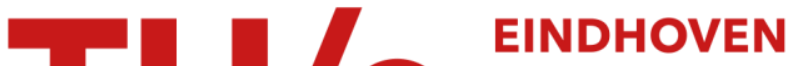 \\ UNIVERSITY OF \\ TECHNOLOGY
}

\section{Nonaxisymmetric Effects in Drop-On-Demand Piezoacoustic Inkjet Printing}

\section{Citation for published version (APA):}

van der Meulen, M. J., Reinten, H., Wijshoff, H., Versluis, M., Lohse, D., \& Steen, P. (2020). Nonaxisymmetric Effects in Drop-On-Demand Piezoacoustic Inkjet Printing. Physical Review Applied, 13(5), [054071]. https://doi.org/10.1103/PhysRevApplied.13.054071

DOI:

10.1103/PhysRevApplied.13.054071

Document status and date:

Published: 28/05/2020

\section{Document Version:}

Publisher's PDF, also known as Version of Record (includes final page, issue and volume numbers)

\section{Please check the document version of this publication:}

- A submitted manuscript is the version of the article upon submission and before peer-review. There can be important differences between the submitted version and the official published version of record. People interested in the research are advised to contact the author for the final version of the publication, or visit the $\mathrm{DOI}$ to the publisher's website.

- The final author version and the galley proof are versions of the publication after peer review.

- The final published version features the final layout of the paper including the volume, issue and page numbers.

Link to publication

\section{General rights}

Copyright and moral rights for the publications made accessible in the public portal are retained by the authors and/or other copyright owners and it is a condition of accessing publications that users recognise and abide by the legal requirements associated with these rights.

- Users may download and print one copy of any publication from the public portal for the purpose of private study or research.

- You may not further distribute the material or use it for any profit-making activity or commercial gain

- You may freely distribute the URL identifying the publication in the public portal.

If the publication is distributed under the terms of Article 25fa of the Dutch Copyright Act, indicated by the "Taverne" license above, please follow below link for the End User Agreement:

www.tue.nl/taverne

Take down policy

If you believe that this document breaches copyright please contact us at:

openaccess@tue.nl

providing details and we will investigate your claim. 


\title{
Nonaxisymmetric Effects in Drop-On-Demand Piezoacoustic Inkjet Printing
}

\author{
Mark-Jan van der Meulen, ${ }^{1, *}$ Hans Reinten, ${ }^{2}$ Herman Wijshoff, ${ }^{2}$ Michel Versluis, ${ }^{1}$ Detlef Lohse $\odot,{ }^{1,3, \dagger}$ \\ and Paul Steen ${ }^{4, \$}$ \\ ${ }^{1}$ Physics of Fluids Group, Max-Planck Center for Complex Fluid Dynamics, MESA+ Research Institute, and \\ J.M. Burgers Centre for Fluid Dynamics, Department of Science \& Technology, University of Twente, P.O. Box \\ 217, 7500 AE Enschede, Netherlands \\ ${ }^{2}$ Océ Technologies, P.O. Box 101, 5900 MA Venlo, Netherlands \\ ${ }^{3}$ Max Planck Institute for Dynamics and Self-Organization, Am Fassberg 17, 37077 Göttingen, Germany \\ ${ }^{4}$ School of Chemical and Biomolecular Engineering, Cornell University, Olin Hall 120, Ithaca, \\ New York 14853, USA
}

(Received 5 October 2019; revised manuscript received 26 January 2020; accepted 14 April 2020; published 28 May 2020)

\begin{abstract}
Drop-on-demand (DOD) inkjet printing is well characterized and a well-studied problem, but nonaxisymmetric effects are typically ignored, while these effects can severely reduce the print-head performance and its stability. In this paper we first review nonaxisymmetric droplet formation originating from geometrical effects. We then focus on the possibility that observed nonaxisymmetry arises from surface instabilities of the meniscus by a Rayleigh-Taylor-like (RT) mechanism. It is shown theoretically that the meniscus can become RT unstable beyond a critical acceleration. A comparison with data extracted from high-speed recordings of the meniscus oscillations show that the critical accelerations are exceeded. Using the time duration that the critical acceleration is exceeded and the maximal growth rate, the extent of growth of the unstable wave is estimated.
\end{abstract}

DOI: 10.1103/PhysRevApplied.13.054071

\section{INTRODUCTION}

Drop-on-demand (DOD) inkjet printing is a wellestablished noncontact material-deposition method, which is best known for usage in the graphical industry. The great advantage of DOD inkjet printing is that material is only deposited where and when it is needed and that no mask is required. This promises a reduction in production time, cost, and waste for a large range of other applications [1]. Well-known fields in which DOD inkjet printing is applied are printed electronics [2,3], organic light-emitting diodes (OLEDS) [4], and organic solar cells [5]. The digital nature of the technique is interesting because on-demand personalized products can be produced. Applications fields that benefit from this property include integrated smart systems, e.g., radio-frequency identification tags [6], and the highly popular three-dimensional (3D) rapid prototyping [7]. The noncontact nature of inkjet printing allows not only applications on flat surfaces but on more arbitrary shaped surfaces as well, which makes it suitable for all kinds of decorating purposes and deposition on fluidic and other highly fragile structures [8].

\footnotetext{
*markjanvdm@gmail.com

$\dagger$ d.lohse@utwente.nl

$\ddagger$ phs7@cornell.edu
}

\section{A. Print resolution}

The print resolution required for graphical inkjet products is bounded by the spatial resolving capacity of the eye (visual acuity), which for optimal eyes and lightning conditions have been found to be 77 cycles per degree [9]. Therefore, the maximal observable resolution depends on the viewing distance; typically a $10 \times 15 \mathrm{~cm}^{2}$ photograph is observed from a distance of $25 \mathrm{~cm}$, which demands an optimal resolution of approximately 900 dots per inch (DPI). Full color prints require multiple dots per pixel, usually four for CMYK prints, which makes the typically found 600 pixels per inch (PPI) acceptable. For many of the upcoming applications in functional printing, however, a much higher resolution is required. Subpicoliter drops are required to obtain printed features finer than 2400 DPI resolution, corresponding to $10-\mu \mathrm{m}$ features. Current commercial 3D printing resolutions are $42 \mu \mathrm{m}$ in the lateral direction and $16 \mu \mathrm{m}$ in height and the resolution has to be further improved to expand on the number of possible applications [10]. Inkjet technology is interesting for the production of multiple organic electronic devices, but for the use in organic thin-film transistors (OTFTs) a further reduction of the scale is required, since the currently obtained $10-\mu \mathrm{m}$ features limit the operational throughput of these devices [11]. Also for maskless lithography production methods, the size of the 
droplets has to be reduced by at least another order of magnitude [12].

The use of MEMS technology allows for a reduction of the nozzle sizes. For MEMS print-head manufacturing accuracy in the $0.5-\mu \mathrm{m}$ range has been reported $[13,14]$. In the graphical industry, nozzle tolerances of $<0.1 \mu \mathrm{m}$ are pursued. The size of the droplets formed, in principle, is comparable to the nozzle size. However, the droplet size can be reduced through the use of actuation pulse modulation $[15,16]$. Here, higher-order modes of the meniscus oscillations are used to eject droplets that are one order of magnitude smaller than the typical nozzle size. Also, pulsed dc voltages have been applied in combination with electrohydrodynamic inkjet printing [17], leading to controlled drop formation with a drop-to-nozzle diameter ratio down to $1: 15$.

\section{B. Deposition accuracy}

While the resolution of a printed product is primarily determined by the droplet size, after impact and solidification, it is also determined by the droplet deposition accuracy [18]. For example, display applications for smart phones require a placement accuracy of approximately $5 \mu \mathrm{m}$ [19]. The deposition accuracy in principle depends on

(a) the accuracy of the movement of the print head with respect to the paper;

(b) the aerodynamic effects on the droplet in flight;

(c) the directional accuracy of jetting;

(d) the drop formation precision and reproducibility.

What effects are the most crucial ones? It has been found, both numerically and experimentally, that the aerodynamic effects of moving paper has a negligible influence on the droplet displacement of $20-\mu \mathrm{m}$ droplets [20,21]. However, smaller drops become increasingly more vulnerable to the surrounding air flow, as they are carried away more easily. In addition, the smallest droplets, i.e., the emerging satellite drops, do not carry enough momentum to reach the substrate (typical distance is around $1 \mathrm{~mm}$ - obviously contact between substrate and print head must be avoided by all means) and can agglomerate on the nozzle plate, which may lead to nozzle failure. And finally, to maintain the material flow throughput (and thus the time it takes) whilst reducing the droplet size, the DOD frequency must be increased, which unfortunately gives less reproducible drop formation [22]. To find out why this can be the case is the objective of this paper.

In this paper we focus on the last two items in above list. Indeed, the deposition accuracy of jetted droplets is strongly affected by nonaxisymmetric drop formation. Figure 1 illustrates some of the well known examples of nonaxisymmetric effects in droplet formation:

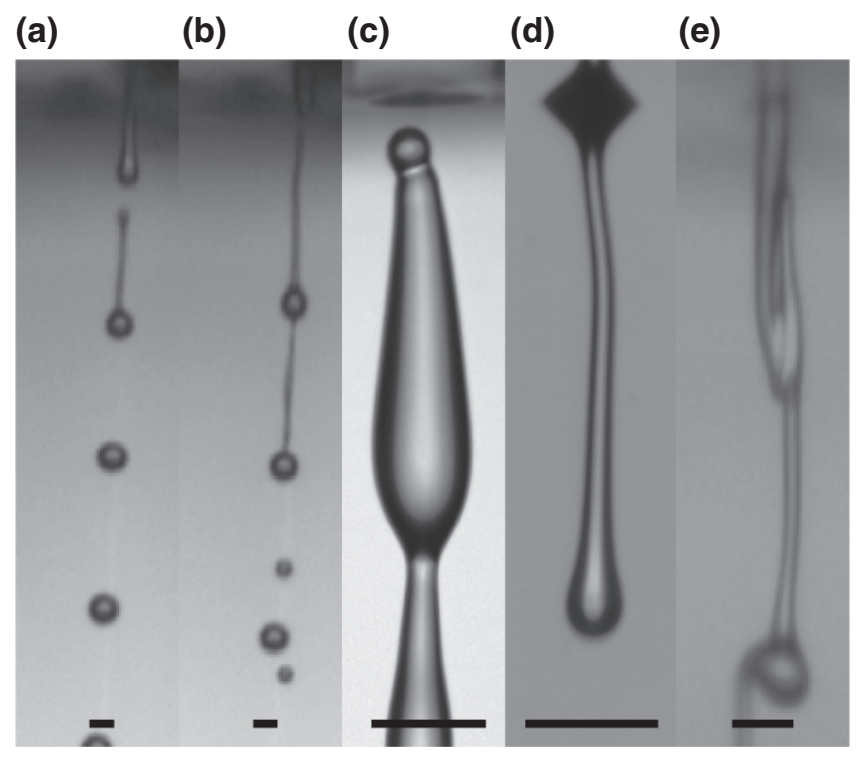

(f)
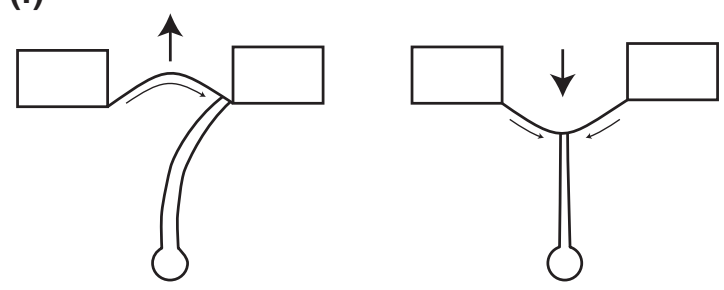

FIG. 1. Observed asymmetrical drop formation in different print heads. The black lines indicate $50 \mu \mathrm{m}$. (a) Jet angle: drops are jetted away from the central axis of the nozzle. (b) Satellite formation: satellite drops are easily jetted away from the main drop. (c) Tail hooking: the tail drop is pulled away towards the nozzle edge. (d) Crooked tail: the droplet tail is pulled to the nozzle wall and pushed back to the center axis causing a deflection in the tail. (e) Sidewards pushed drops: when a droplet is jetted shortly after an asymmetrical drop, the new drop is strongly affected. (f) Tail hooking: the tail attached to the meniscus has a preference to sweep to one side of the nozzle upon retraction, and is centered during extortion of the meniscus outside of the nozzle.

(a) Figure 1(a) displays a problem with jetting directionality also referred to as jet angle, where the droplet is jetted at an angle with respect to the direction parallel to the nozzle plate. These are problematic for inkjet devices since the droplet is not deposited at the target site on the substrate. Only constant jet angle in the direction of the motion of the print head, can be corrected for using additional time delays. But often this is not the case and the jet angle also changes during the jetting process, which gives rise to large deposition inaccuracies $[23,24]$.

(b) Asymmetrical drop formation, such as those shown in Fig. 1(b), gives rise to an increased generation of satellite drops [25]. Satellite drops are typically formed from the Rayleigh-Plateau breakup of thin droplet tails. In an 
axisymmetrical situation, the trailing satellites can easily merge with the following drops due to their different velocities. However, for the nonaxisymmetrical case, the satellites can be directed along a different trajectory than the main drops, reducing their chance to be captured.

(c) Upon retraction of the meniscus into the nozzle, the connected droplet tail is often observed to sweep to one side of the nozzle, making the subsequent drop-formation process nonaxisymmetric, see Fig. 1(c). If the tail of the droplet is not yet pinched off from the meniscus at this stage, the deflection causes so-called tail hooking [26,27]. This process can be compared to a string under tension, confined at one side to a spherical cap. By sweeping the tail to one side, it shortens and releases its stress, as indicated in Fig. 1(f). In a later stage, where the meniscus is outside the nozzle, the preferred position of the tail is at the apex of the meniscus. Thus, the particular orientation of the droplet tail depends on the exact moment of pinch off. When the tail pinches off from the meniscus at the moment when the meniscus retracts, the tail droplet and possible satellite droplets are catapulted in a sideways direction.

(d) In case the tail droplet does not detach from the meniscus, it restores to the center position resulting in a crooked tail, see Fig. 1(d). The main droplet is not strongly affected by the occurrence of an asymmetry in the tail, due to the large difference in inertia. However, even jet angles down to nozzle plate $0.1^{\circ}$ cause a relevant and measurable change in drop deposition position.

(e) At higher DOD frequencies, the droplet is not easily pinched off from the meniscus, before the following droplet is produced. When the first droplet is not perfectly symmetric, the next droplet flows alongside it, enhancing the asymmetry. This can give rise to a zig-zag dropformation mode with higher-order or chaotic deviations from the symmetric drop formation, see Fig. 1(e).

\section{External causes of the asymmetries and their relevance}

The asymmetries in real inkjet devices are often initiated by various asymmetrical effects near the nozzle and the origin of these effects are suspected to trigger the deviation of jetting with respect to the nozzle axis, see the set of illustrations in Fig. 2. Causes of the asymmetry include the following.

(a) A very relevant problem are asymmetries in the print head itself and in the nozzle geometry, Fig. 2(a). These include positional or directional misalignments of the print-head channel with respect to the nozzle.

(b) Moreover, dirt- or dust-particle agglomerates stuck in the nozzle area can form an obstacle that redirects the fluid flow, see Fig. 2(b).

(c) One further problem causing asymmetries is the wetted nozzle plate, which typically is not perfectly
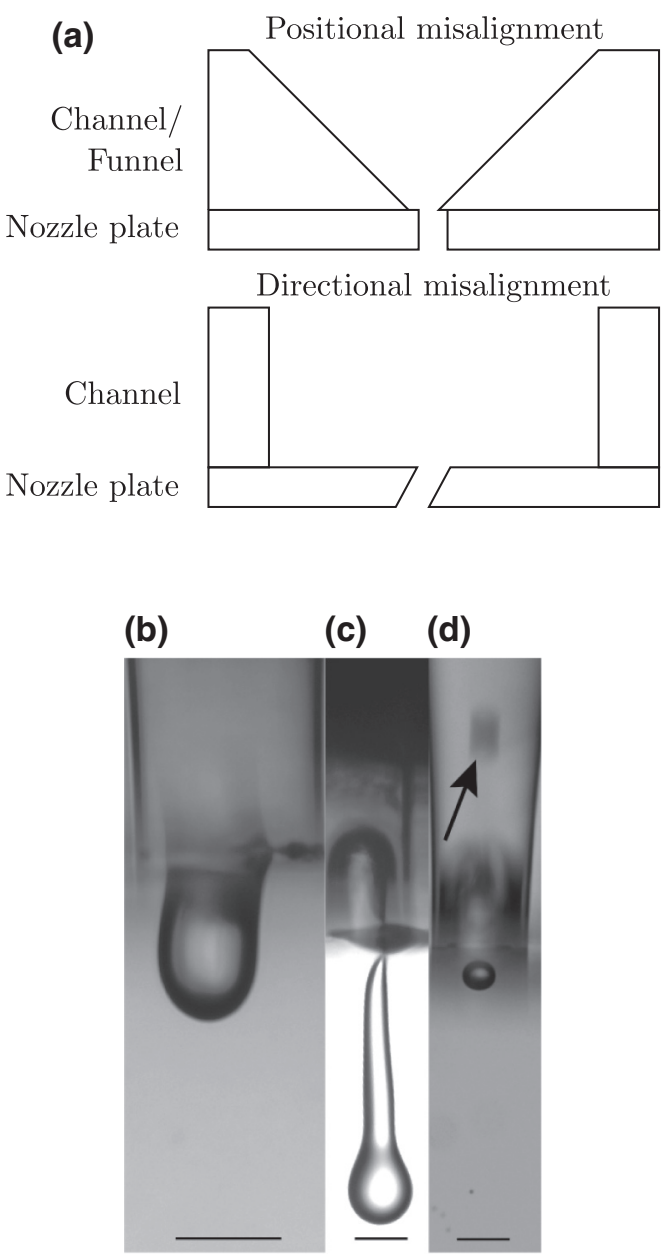

FIG. 2. Asymmetric drop formation by geometrical origin. (a) Print-head- and nozzle-geometry asymmetries. (b) Dirt in the nozzle, causing the drop to deflect. (c) Asymmetrically wetted nozzle plate easily results in coalescence of the tail drop with the wetted area and deflection of the droplet. (d) Bubble entrapped off center inside the nozzle, indicated with in arrow, deforming the acoustic actuation wave asymmetrically. The black lines indicate $50 \mu \mathrm{m}$.

symmetric around the nozzle entrance, due to chemical and geometric heterogeneities. Therefore, the meniscus is not fully pinned to the nozzle edge but at some point may coalesce with the wetted area around the nozzle. Thus, the asymmetry initiated by the location of the wetted area then results in an asymmetric ejection from the nozzle, see Fig. 2(c).

(d) Finally, bubbles entrapped inside the inkjet nozzle can grow due to rectified diffusion [28,29]. When these bubbles become too large, see e.g., Fig. 2(d) they can absorb the applied pressure wave, which leads to nozzle failure [30,31], as the pressure increase is not sufficient for droplet jetting. However, even a smaller bubble, one that 
can freely move within the nozzle, may cause an asymmetric effect to the pressure wave directed towards the meniscus.

In the last few years there has been a growing interest in nonaxisymmetrical drop formation. Kwon et al. [32] reported on the axiswitching of a drop asymmetry in time resulting from an elliptical nozzle. Castrejón-Pita et al. [33] showed that mechanical defects outside the nozzle plate have a negligible effect on the drop formation, but when the defect intrudes into the nozzle flow strong deflection angles are introduced. Recently, Harlen et al. [27] showed experimentally and numerically that droplets detach asymmetrically from nozzles, which are under an angle with respect to the nozzle plate.

\section{Asymmetry caused by intrinsic instabilities}

While the nonaxisymmetric drop formation in the above work has a clear geometrical origin, which in several cases was intentionally imposed by the researchers, in this paper we hypothesize that nonaxisymmetric drop formation can also be the result of unstable asymmetrical surface modes at the meniscus interface.

Indeed, the enormous acceleration applied to the fluid across the inkjet meniscus (approximately $10^{5} \mathrm{~g}$ ) during the printing process, allows instabilities to grow against surface tension for much smaller waves than in the classical Rayleigh-Taylor context [34,35]. These accelerations approach those generated by shock waves, known to trigger the Richtmyer-Meshkov (RM) instability [36]. The $\mathrm{RM}$ is closely related to the RT instability. Whether the fluid acceleration be constant (classical RT), impulsive (RM), or time varying (this paper), the instability mechanisms (and theory) are essentially the same so we hereafter refer to them simply as "RT." Essential to our modeling as an RT instability is that the period of time-varying acceleration is long compared to the time for instability growth. That is, the timescales are assumed to separate. The RT theory gives rise to symmetric and antisymmetric surface modes, and can therefore have large consequences for stable inkjet printing.

\section{E. Structure of paper}

In this paper, to evaluate the RT instability in the inkjet context, we scale the instability theory with the relevant parameters involved with the meniscus motion in the nozzle. The analysis leads to an admissible and unstable regime, in which inkjet meniscus motions can be placed (Sec. II). We then experimentally extract the acceleration of the meniscus interface in a transparent inkjet system and place these experiments in context of the instability theory (Sec. III). As we show in Sec. IV, with the analysis one can determine whether waves on the inkjet meniscus are unstable and how large they can grow during an inkjet actuation

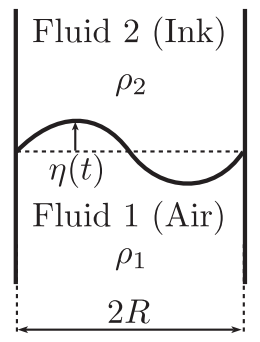

FIG. 3. Rayleigh Taylor instability.

cycle. The paper ends with conclusions (Sec. V), in which we, in particular, present a detailed list of questions, which can be used by the practitioner to decide on whether RT instabilities are relevant in the problem at hand.

\section{THEORETICAL APPROACH TO THE MENISCUS INSTABILITY}

Accelerating a dense fluid into a less dense fluid causes surface instabilities when the inertial forces overcome the restoring surface tension. For inkjet nozzles of characteristic scale (diameter) $2 R$ we consider an ink-air interface having surface tension $\gamma$ with fluid 1 arranged below a fluid 2 with densities $\rho_{1}$ and $\rho_{2}$, see Fig. 3 . The classical RT instability occurs when gravity accelerates from heavy into light fluid. We adapt the classical RT results to surface-wave disturbances in inkjet nozzles. For nozzles, the gravitational acceleration is replaced by the acceleration of the mean liquid motion within the nozzle. This acceleration is heavy into light fluid during the in stroke with a maximum when the meniscus position is most inward, much like the extreme position during the pendulum up swing. At most, viscosity slows growth rates of unstable disturbances and can be neglected altogether for Ohnesorge number Oh $\equiv \mu_{2} / \sqrt{\rho_{2} \gamma R} \ll 1$, with $\mu_{2}$ the viscosity of liquid 2 . Oh $\ll 1$ for inks studied in this paper.

For classical RT dispersion on a flat interface of infinite extent, the growth rate $s$ relates to the wave number $k$ by

$$
s^{2}=\frac{k\left[g\left(\rho_{2}-\rho_{1}\right)-\gamma k^{2}\right]}{\rho_{2}+\rho_{1}},
$$

where $g$ is the gravitational acceleration. From the dispersion relation it follows that the perturbation $\eta(t) \sim e^{\text {st }}$ is always (neutrally) stable for $\rho_{2}<\rho_{1}$ since then, $s^{2}<0$, in which case only traveling waves occur, and is unstable in time $t$ for allowed combinations $k_{m, n} r$ (where $r$ is the radial coordinate) when the Bond number

$$
B_{o} \equiv \frac{g r^{2}(\Delta \rho)}{\gamma}>(k r)^{2},
$$

with $\Delta \rho \equiv \rho_{2}-\rho_{1}$, since then $s^{2}>0$, in which case $s=$ $\pm \sqrt{s^{2}}$, and the positive root yields exponential growth. 


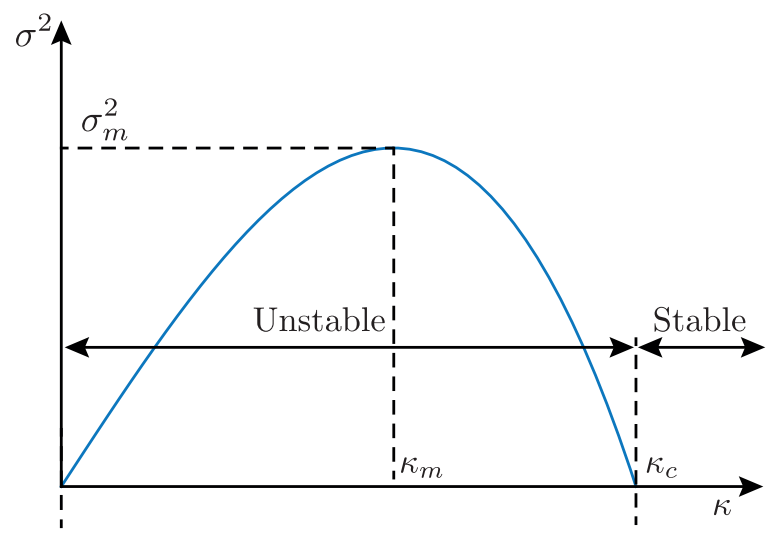

FIG. 4. Classical RT dispersion relation (scaled). $\kappa$ is the wave number of the distortion and $\sigma$ the growth rate.

In preparation for evaluating the stability of inkjet menisci relative to admissible and growing disturbances, we anticipate confinement of the disturbance by the nozzle and replace $r$ by $R$, the nozzle radius. Larger disturbances cannot fit, and therefore are inadmissible. We also replace $g$ by an acceleration $a$, which is the mean meniscus acceleration of heavy into light fluid. (We retain $g$ as the acceleration reference scale.) In summary, the classical RT dispersion relation (1) can be nondimensionalized to

$$
\sigma^{2}=\kappa\left(B-\kappa^{2}\right),
$$

by the scaling choice

$$
\kappa \equiv k R, \quad \sigma \equiv s \tau_{c}, \quad \text { and } \quad B \equiv \lambda B_{o} .
$$

Here $\tau_{c}$ is the inertial and capillary timescale and $\lambda$ the ratio of accelerations, defined as

$$
\tau_{c} \equiv \sqrt{\rho_{2} R^{3} / \gamma} \quad\left(\rho_{2}>>\rho_{1}\right), \quad \lambda \equiv a / g
$$

The scaled dispersion relation is plotted in Fig. 4.

From the scaled dispersion relation we deduce the following parameters, indicated in Fig. 4. The critical wave number $\kappa_{c}$ for stability is

$$
\kappa_{c}=\sqrt{B} .
$$

The wave number $\kappa_{m}$ of maximal growth is

$$
\kappa_{m}=\sqrt{\frac{B}{3}}
$$

and the maximal growth rate $\sigma_{m}$ is given by

$$
\sigma_{m}^{2}=\frac{2}{3 \sqrt{3}} B^{3 / 2}
$$

In Fig. 5 the unstable RT regime is plotted in a diagram of wave number against applied acceleration. All waves

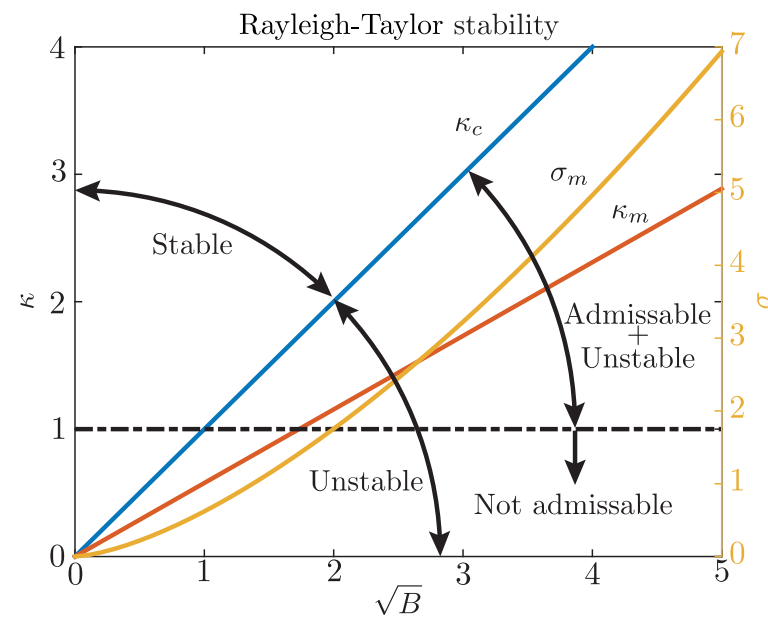

FIG. 5. Window of instability (black arrows) for a finite meniscus. For a given wave number $\kappa$, scale at left, surface waves on the fluid meniscus grows for large enough acceleration $B$, the "Unstable" window. For these, $\kappa_{m}$ indicates the wavelength of maximum growth $\sigma_{m}$, scale at right. Wave numbers must also be such that the disturbance fits into the nozzle cross section. Waves with $\kappa<1$ (dashed) do not fit, labeled "Not admissible." Potentially observable disturbances fall in the "Admissible + Unstable" window.

with wave number smaller than $\kappa_{c}$ are unstable, no matter the extent of the interface, providing the upper limit to the window of instability. However, only wave numbers $\kappa>1$ can accommodate the nozzle constraint, called admissible wave numbers, providing the lower limit. Surface waves never grow for accelerations $\sqrt{B}<1$. The "admissible and unstable" regime widens for larger accelerations, allowing more wavelengths to grow. The line $\kappa_{m}$ indicates the fastest growing wave number at a given acceleration $B$, and curve $\sigma_{m}$ gives the corresponding growth rate. A further discussion of wave-number selection is given in the next section.

\section{A. Wave-number selection}

Wave-number selection occurs when restricting to a bounded domain, here a nozzle of radius $R$. The wave numbers are selected by the boundary condition at the nozzle. The general lower admission criterion, indicated in Fig. 5, is set by the demand that at least one wavelength of the disturbance fits the container boundary,

$$
\kappa=k * R>1 .
$$

Smaller values of $\kappa$ are not admissible for any boundary condition or nozzle geometry with radius $R$.

For a cylindrical container and negligible Oh, the general solution of the RT wave equation for the deflection amplitude $\eta(t)$ of the flat meniscus reads [37]

$$
\eta(t)=\epsilon J_{m}\left(k_{m n} r\right) e^{i m \theta+\mathrm{st}},
$$




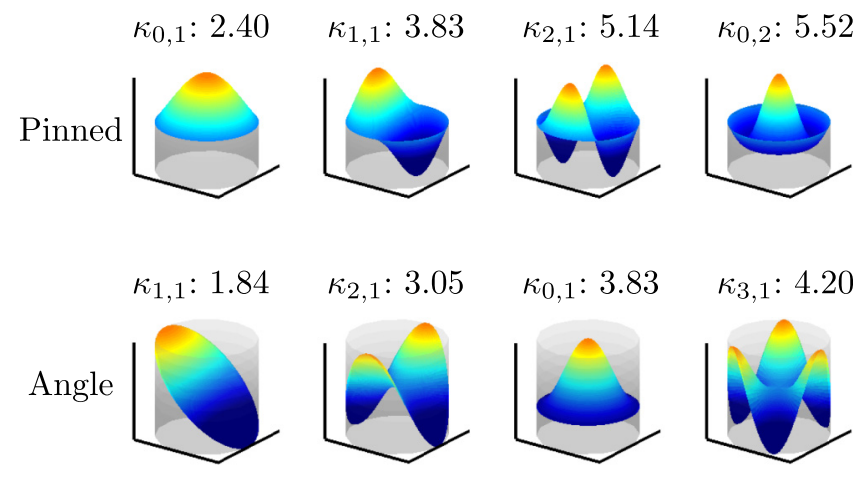

FIG. 6. Wave numbers $\kappa$ and corresponding disturbance shapes are selected by fitting the solutions, Eq. (10), to the boundary condition at the nozzle wall. The selected shapes are plotted for pinned (Pinned) and for constant $90^{\circ}$ contact-angle (Angle) boundary conditions.

where $\epsilon$ is an initial disturbance amplitude, $J_{m}$ is the Bessel function of the first kind and $k_{m n}$ the wave numbers of the perturbations. This solution was obtained assuming potential flow, valid for negligible Oh. Given the nozzle radius, the wave numbers are selected through the edge boundary condition. For a pinned meniscus at the nozzle edge or wall, $J_{m}\left(\kappa_{m n}\right)=0$. A commonly used alternative is a moving contact line with a constant contact angle of $90^{\circ}$ (perfect slip), $J_{m}^{\prime}\left(\kappa_{m n}\right)=0$. The selected wave numbers correspond to symmetric and asymmetric meniscus eigenmodes as illustrated in Fig. 6. The exact wave number depends on the exact boundary condition at the nozzle, but the least restrictive boundary condition and hence the largest wavelength (smallest wave number) belongs to the case of perfect slip, the "natural boundary condition" in the language of the variational calculus. In summary, wavenumber selection occurs regardless of wettability details.

\section{B. Instability estimates}

Figure 5 provides key information for assessing observability of meniscus instability in inkjet nozzles. For example, one learns that only waves with wave numbers $\kappa>1$ fit inside of inkjet nozzles and hence that the acceleration of the meniscus must at least be $\sqrt{B}>1$ to excite admissible waves.

When the meniscus in an inkjet nozzle is subjected to a specific acceleration $B, \sqrt{B}>1$, all waves within the admissible and unstable regime can grow. The exact unstable shape depends on the local boundary condition at the nozzle wall. For various boundary conditions, the stability of the selected shapes shown in Fig. 6 determined using Eq. (6). The accelerations $B$ above which the selected shapes become unstable are indicated in Table I.

The maximal growth rate $\sigma_{m}$ of an unstable disturbance can be used to judge observability, as follows. The potential end size of a growing disturbance depends not only on how fast it grows but also on how long it has to grow.
TABLE I. Acceleration $B$ above which the selected wave numbers $\kappa_{m, n}$ grow, for contact-line-pinned and constant-angle boundary conditions. Pairs $\left(B, \kappa_{m, n}\right)$ fall on the $\kappa_{c}$ curve, Fig. 5, illustrating how wave number is quantized by selection. Acceleration factor $\lambda=B / B_{0}$ with $B_{0}=8.4 * 10^{-5}$ for the experimental system studied.

\begin{tabular}{lcccc}
\hline \hline & \multicolumn{5}{c}{ Pinned } \\
& $\kappa_{0,1}$ & $\kappa_{1,1}$ & $\kappa_{2,1}$ & $\kappa_{0,2}$ \\
\hline$B$ & 5.76 & 14.7 & 26.4 & 30.5 \\
& & Angle & & \\
& $\kappa_{1,1}$ & $\kappa_{2,1}$ & $\kappa_{0,1}$ & $\kappa_{3,1}$ \\
\hline$B$ & 3.38 & 9.3 & 14.7 & 17.6 \\
\hline \hline
\end{tabular}

Growth is limited to the in-stroke phase of the meniscus motion during an inkjet actuation cycle. The duration that the acceleration exceeds the critical acceleration is called the gestation period $\tau_{p}$. Exponential growth occurs for a maximum time $\tau_{p} / \tau_{c}$ at a maximum (scaled) rate of $\sigma_{m}$, to yield an amplification on order $\exp \left(\sigma_{m} \tau_{p} / \tau_{c}\right)$.

Next, experiments with a transparent inkjet nozzle jetting water are performed to relate the fluid motion in inkjet print heads to the RT theory. From the experiments we obtain the acceleration of the fluid meniscus in the nozzle and also the duration for which accelerations are maintained. Using this data we estimate the growth of unstable waves during these gestation periods.

\section{EXPERIMENTAL INVESTIGATION}

\section{A. Print head}

Experiments are performed using an AD-K-501 Autodrop Pipette of Microdrop Technologies. This print head is built from a single nozzle glass tapered nozzle and is actuated with a cylindrical piezo element. The glass nozzle is optically transparent, which allows for inspection of the meniscus movement within the nozzle. The $25-\mu \mathrm{m}$ radius nozzle is connected to a long tapered channel, therefore the resonance frequency of this print head is predicted by the wave-guide theory [38] and is determined by the traveling time of the pressure wave through the microchannel. This type of print head is typically used for water-based inks and in the current study we use water, which is purified using a $20-\mu \mathrm{m}$ filter. From the oscillation frequency of the meniscus, the resonance frequency of this print head is estimated to be $7 \mathrm{kHz}$.

\section{B. Visualization setup}

For reproducible drop formation, time-resolved singleflash illumination can be used to obtain high-resolution stroboscopic information on the drop formation [39]. The droplets are recorded with a shadowgraphic imaging technique, see Fig. 7. For every image recorded with PCO 


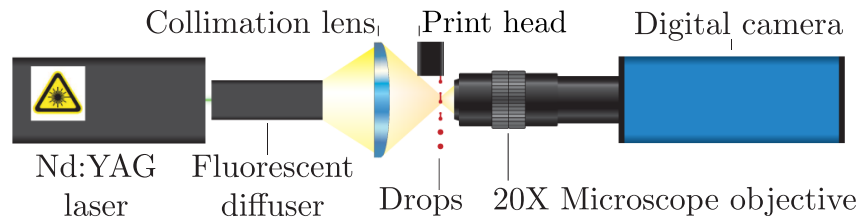

FIG. 7. Experimental setup: a 7-ns Nd:YAG laser pulse is converted to noncoherent fluorescence light for illumination by a fluorescent diffuser. Stroboscopic imaging with incremental delays with respect to the actuation pulse make up a full recording of the meniscus movement.

Sensicam QE, a fresh droplet is produced while an incremental delay of the recording with respect to the drop actuation is imposed. The ejected micro droplets typically move with a velocity of $10 \mathrm{~m} / \mathrm{s}$, therefore to prevent motion blur, the illumination time has to be set smaller than $30 \mathrm{~ns}$ for a typical resolution of $300 \mathrm{~nm} /$ pixel. The illumination is provided by aNd:YAG laser pulse (pulse length of $7 \mathrm{~ns}$ at a wavelength of $532 \mathrm{~nm}$ ) and a fluorescent diffuser (LaVision) making use of the illumination by laser-induced fluorescence technique (ILIF) [40].

\section{Analysis of meniscus movement from recorded image sequences}

The fluid meniscus is traced both inside and outside of the nozzle exit. Most of the meniscus movement and the highest accelerations take place within the nozzle. The position of the meniscus is determined by finding the inflection point of the intensity profile across the fluid boundary. Figure 8 shows typical traces of the meniscus during an actuation cycle, recorded with $0.1 \mu$ s increasing delay time steps. The meniscus first is pulled back into the nozzle, where it reaches its deepest position around $t=45 \mu \mathrm{s}$. At around $t=70 \mu \mathrm{s}$ the meniscus reaches the nozzle exit after which the droplet is formed. And finally at $t=140 \mu \mathrm{s}$ the meniscus is retracted back into the nozzle. Then the cycle repeats with a period of oscillation of approximately $175 \mu \mathrm{s}$. On top of this global movement a higher frequency meniscus movement is observed, appearing as dents and bumps in the position trace.

The acceleration is extracted from the meniscus position. The experimental noise, however, does not allow for the calculation of the second derivative of the positiontime data directly and the data is smoothed using low-pass frequency filtering. The time steps of the time-resolved series are $0.1 \mu \mathrm{s}$, giving noise in the $\mathrm{MHz}$ range, the meniscus moves typically with order $10 \mathrm{kHz}$, and a more rapid oscillation is observed on top of the main meniscus movement with a typical frequency order of $100 \mathrm{kHz}$. Applying low-pass filters with a cutoff frequency $40 \mathrm{kHz}$ and 140 $\mathrm{kHz}$ gave optimal results while maintaining the separation of scales of the acceleration of the meniscus.

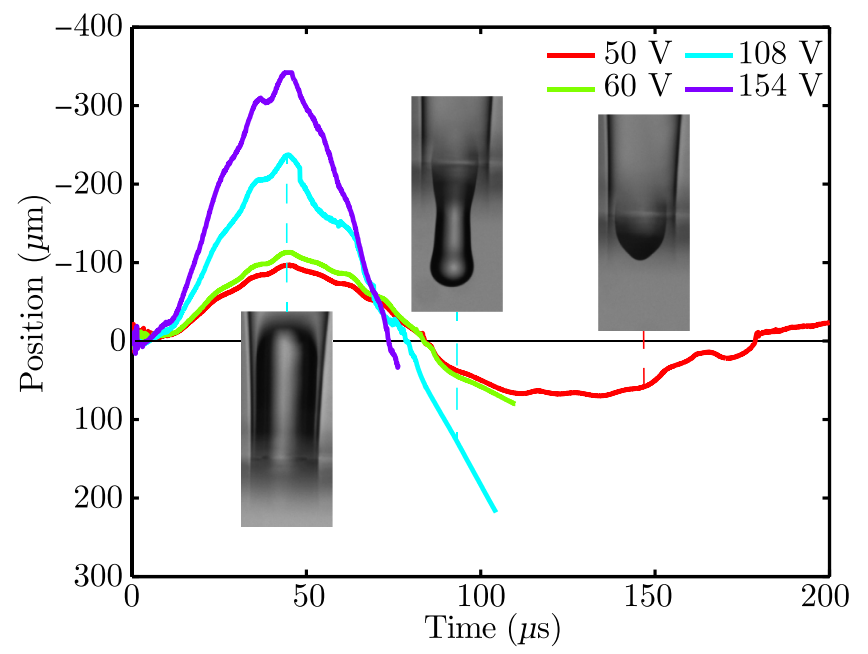

FIG. 8. Meniscus traces for different actuation voltages in the Microdrop print head, see Sec. III A. Photos of menisci at corresponding times are shown. A negative-position value indicates a position inside the nozzle. The curves end at droplet pinch off. For the $50-\mathrm{V}$ actuation the meniscus oscillates within the nozzle. For actuations of $60 \mathrm{~V}$ and higher a droplet is ejected. Peak acceleration of heavy into light coincides with the most inward position, as in an upward pendulum swing.

\section{Experimental results}

Driving conditions are plotted in Fig. 8 for experiments with driving voltages of 50,60,108, and $154 \mathrm{~V}$. The first driving voltage did not create sufficient pressure to form
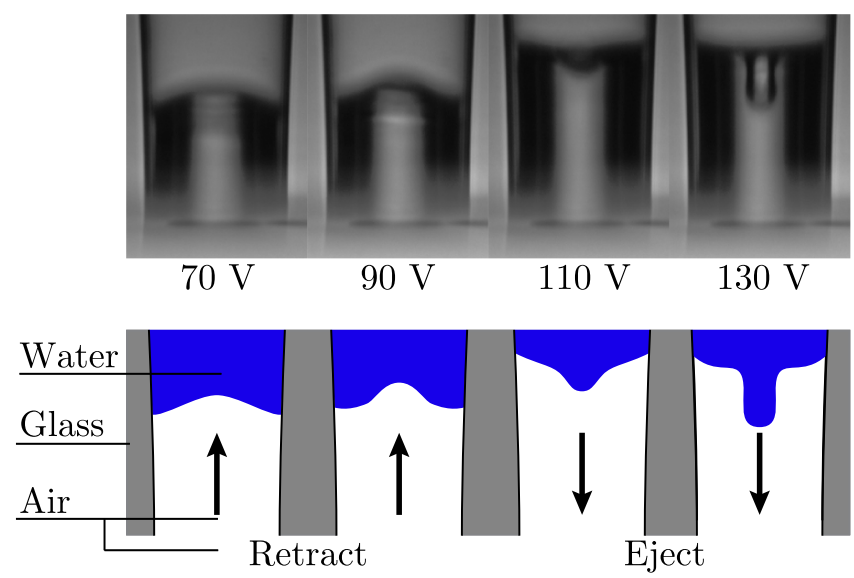

FIG. 9. Upper row: experimentally observed meniscus shapes in the transparent nozzle for four different actuation voltages. Lower row: illustrative drawings of the four cases. For lower actuation voltages the meniscus shapes resemble the (symmetrical) normal mode $\kappa_{0,1}$, Fig. 6 , however for higher actuations the meniscus deforms to a shape resembling the (symmetrical) $\kappa_{0,2}$ mode, where a protrusion is formed, Fig. 6 . This protrusion oscillates with a higher temporal frequency around the apex of the meniscus. Arrows upward (downward) indicate the in-stroke (out-stroke) phase. 

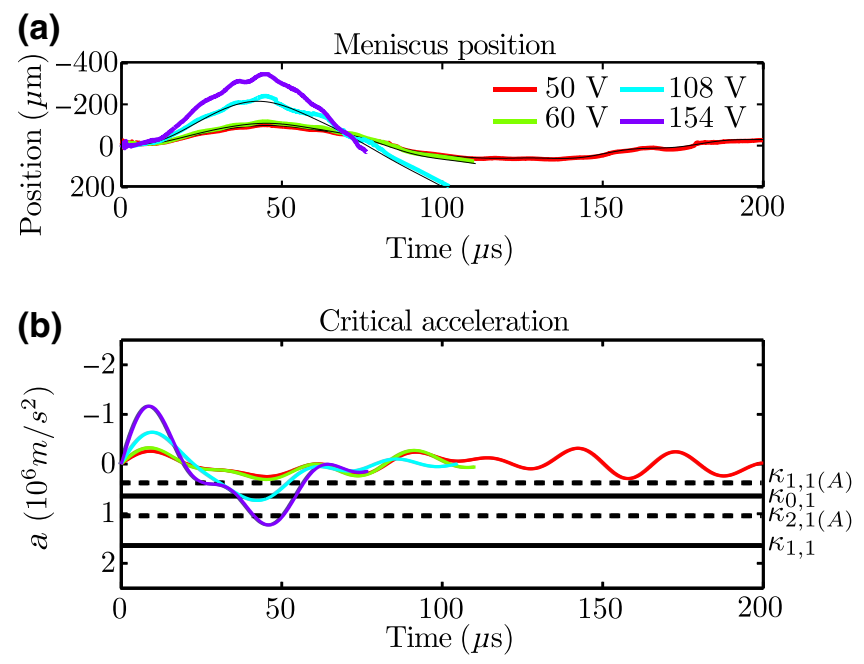

FIG. 10. Meniscus position and acceleration traces, and RT thresholds using the 40-kHz low-pass filter: (a) filtered meniscus position for various actuation voltages (colored), meniscus position reconstructed from filtered accelerations (black); (b) filtered accelerations for voltages (colored), with RT-instability thresholds corresponding to the various $\kappa_{m, n}$ indicated, for a pinned (solid line) and constant angle [dashed line, subscript $(A)$ ] boundary condition. Just as in Fig. 8, here and in the next figure the curves end at droplet pinch off. For the weak actuation of $50 \mathrm{~V}$, there is no pinch off.

a droplet, but instead the meniscus is oscillating around the nozzle exit. For a $60-\mathrm{V}$ driving pulse a droplet is formed, but the velocity of the resulting droplet is very low $(<0.2 \mathrm{~m} / \mathrm{s})$. For the higher voltages droplets are ejected.

For low actuation voltages the meniscus shape resembles the meniscus mode $\kappa_{0,1}$ of Fig. 6. For actuation voltages larger than $90 \mathrm{~V}$, however, the observed meniscus shape resembles the mode $\kappa_{0,2}$, where a protrusion in the center area of the meniscus oscillates with a higher frequency around its rim as shown in Fig. 9. When the actuation voltage exceeds $110 \mathrm{~V}$ a smaller droplet is ejected from this central area, even though the global meniscus movement is still at the $7-\mathrm{kHz}$ frequency.

The main meniscus movement, using the $40-\mathrm{kHz}$ lowpass filter is plotted in Fig. 10. In the lower part of the figure the acceleration is plotted together with the critical acceleration thresholds. The thresholds for the constant contact angle are indicated with the suffix ${ }_{(A)}$. For the actuation with voltages $108 \mathrm{~V}$ and higher, the acceleration thresholds for the axisymmetric $\kappa_{0,1}$ are exceeded when the meniscus accelerates inward. First around $8 \mu \mathrm{s}$ after the actuation of the print head, and again when it is accelerated back outwards of the nozzle at time $40 \mu \mathrm{s}$. The RT instability sets in when the fluid with higher density is accelerated into the fluid with lower density, i.e., when the meniscus is instantaneously stationary as it transitions from pull back to push out. The acceleration crossed the threshold for
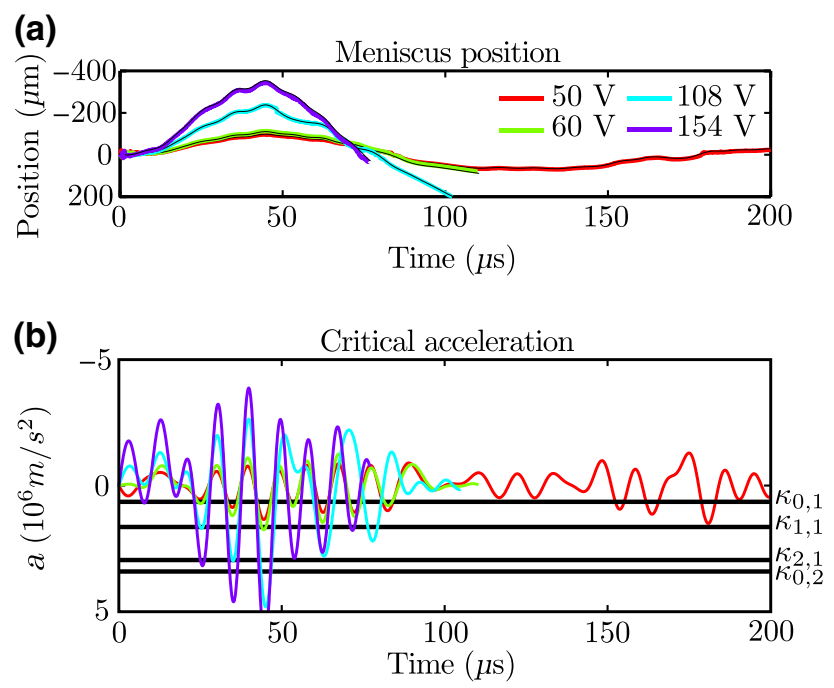

FIG. 11. Meniscus position and acceleration traces, and RT thresholds using the $120-\mathrm{kHz}$ low-pass filter: (a) filtered meniscus position for various actuation voltages (colored), meniscus position reconstructed from filtered accelerations (black); (b) filtered accelerations for voltages (colored), with RT-instability thresholds (solid line) corresponding to the various $\kappa_{m, n}$, indicated, for the pinned case only.

longer than $15 \mu \mathrm{s}$. For the pinned meniscus the accelerations from the main meniscus movement never cross the acceleration thresholds for the asymmetrical modes. For some lower actuations, even when drops are ejected, none of the pinned thresholds are exceeded during actuation of the print head. For the nonpinned meniscus the first instability threshold is for the asymmetrical mode $\kappa_{1,1(A)}$ at $a=$ $0.39 \times 10^{6} \mathrm{~m} / \mathrm{s}^{2}$, which is crossed for all droplet actuations and close to the actuation acceleration where a droplet is just formed.

In Fig. 11, the same data are shown, but now with low-pass filtering at $140 \mathrm{kHz}$, keeping the faster oscillations. These accelerations are approximately four times faster and for most accelerations the $\kappa_{0,1}$ thresholds are exceeded. Also the 50-V actuation, which did not eject a droplet, exceeded this threshold. The time spans during which the accelerations are above the threshold, are now shorter, 5-6 $\mu \mathrm{s}$.

\section{RESULTS AND DISCUSSIONS}

From the timescale $\tau_{c}$, Eq. (5), one finds the characteristic growth time for RT waves of $\tau_{c}=14.7 \mu$ s for our experiments using water and a $25-\mu \mathrm{m}$ radius. This growth time is an order of magnitude shorter than the typical oscillation period of the meniscus, from the 7-kHz actuation frequency. This separation of timescales allows RT waves to potentially grow in the inkjet nozzle, consistent with the meniscus mean motion serving as the carrier for the RT 
TABLE II. Growth table for meniscus motions, $40 \mathrm{kHz}$ filtered: accelerations $a$ (column 2) and corresponding gestation times $\tau_{p}$ (column 5) from experiment, Fig. 10(b), combine with predicted growth rate $\sigma_{m}$ (column 6), from $B$ and Fig. 5, to ultimately yield the amplification factor $\exp \left(\sigma_{m} \tau_{p} / \tau_{c}\right)$ (column 8). Columns 3 and 4 give the prior column values scaled differently.

\begin{tabular}{lccccccc}
\hline \hline Voltage $(\mathrm{V})$ & $a\left(\times 10^{6} \mathrm{~m} / \mathrm{s}^{2}\right)$ & $\lambda\left(\times 10^{4}\right)$ & $\sqrt{B}$ & $\tau_{p}(\mu \mathrm{s})$ & $\sigma_{m}^{2}$ & $\sigma_{m} \tau_{p} / \tau_{c}$ & $\exp \left(\sigma_{m} \tau_{p} / \tau_{c}\right)$ \\
\hline 50 & 0.25 & 2.6 & 1.46 & 0 & 1.2 & 0 & 1 \\
60 & 0.31 & 3.2 & 1.63 & 0 & 1.66 & 0 & 1 \\
108 & 0.72 & 7.3 & 2.48 & 21.2 & 5.89 & 3.52 & 33.7 \\
159 & 1.23 & 12.5 & 3.24 & 31.2 & 13.15 & 7.73 & 2284.5 \\
\hline \hline
\end{tabular}

instability and supplying the necessary acceleration during the in stroke of actuation, Figs. 10(b), 11(b).

\section{A. Growth extent}

The next consideration is the growth extent of unstable waves in the microdrop inkjet nozzle. The maximal measured accelerations and corresponding $\lambda$ values from Fig. 10 are indicated in Table II. The growth time for the waves in the experiment is the gestation period $\tau_{p}$, the duration that the acceleration is above the critical acceleration of the most unstable mode under cylindrical confinement, i.e., $\kappa_{1,1(A)}$ from Fig. 10(b). The maximal growth rate is $\sigma_{m}^{2}=2 /(3 \sqrt{3}) B^{3 / 2}$, where $B=\lambda * B_{o}$, e.g., Fig. 5. An amplification factor is the final result, determined from the product of gestation time and growth rate through

$$
\exp \left(\sigma_{m} \tau_{p} / \tau_{c}\right)
$$

All values are reported in Table II. We find that for the actuations of 50 and $60 \mathrm{~V}$ that the growth extent is 1 . For an actuation of $154 \mathrm{~V}$, we find that the growth extent is 2285 , which is the factor that the wave may grow during the gestation period.

The same evaluation is performed for the experiments indicated in Fig. 11, and the results are indicated in Table III. The motion of the higher frequency component of the meniscus, experiences much higher accelerations $a_{m}$, up to $6 \times 10^{6} \mathrm{~m} / \mathrm{s}^{2}$. In all performed experiments, even those in which no drops are ejected, the acceleration crosses the critical acceleration for the shape with $\kappa_{1,1}$. The gestation periods $\tau_{p}$ are much shorter, however, leaving the waves less times to grow. Consequently, the maximal growth extent for this motion is lower than that found for the $40 \mathrm{kHz}$ low-passed filtered motion. The latter is referred to as the "mean meniscus motion." In summary, for the inkjet formation process studied here, there is considerable evidence that the mean meniscus motion yields accelerations that can trigger RT instabilities. Moreover, relevance of these instabilities depends on how long the instabilities have to grow, the gestation period. Measured gestation periods yield predictions for the observations of drop formation or not.

\section{B. Discussion}

This research studies nonaxisymmetry caused by intrinsic and extrinsic sources and, regarding intrinsic sources, whether surface instabilities of the meniscus by the RT mechanism can play a role in asymmetries in inkjet systems. Before we come to our final conclusions, we address some remaining issues.

Whenever oscillatory forcing generates disturbances to a free meniscus, the possibility of the parametric excitation arises. The Faraday problem is the prototype for parametric meniscus excitation $[41,42]$. The signature response of a parametric excitation is subharmonic, although both subharmonic and harmonic responses are possible [43]. In our drop-on-demand context, parametric excitation is certainly possible but a number of features [44] favor the RT model, which we follow.

The Microdrop print head has a tapered nozzle, which means that the nozzle radius increases going further inside the nozzle, which decreases the acceleration threshold upon retraction of the meniscus. Finally, this print head also exhibits the tail-hooking phenomenon as depicted in Fig. 1(c). Upon retraction of the meniscus back into the nozzle the attached tail sweeps to one side of the meniscus, creating an asymmetrical feature in the drop formation.

TABLE III. Growth table for meniscus motions, $120 \mathrm{kHz}$ filtered: accelerations $a$ (column 2) and corresponding gestation times $\tau_{p}$ (column 5) from experiment, Fig. 10(b), combine with predicted growth rate $\sigma_{m}$ (column 6), from $B$ and Fig. 5, to ultimately yield the amplification factor $\exp \left(\sigma_{m} \tau_{p} / \tau_{c}\right)$ (column 8).

\begin{tabular}{lccccccc}
\hline \hline Voltage $(\mathrm{V})$ & $a\left(\times 10^{6} \mathrm{~m} / \mathrm{s}^{2}\right)$ & $\lambda\left(\times 10^{4}\right)$ & $\sqrt{B}$ & $\tau_{p}(\mu \mathrm{s})$ & $\sigma_{m}^{2}$ & $\sigma_{m} \tau_{p} / \tau_{c}$ & $\exp \left(\sigma_{m} \tau_{p} / \tau_{c}\right)$ \\
\hline 50 & 1.35 & 13.8 & 3.40 & 3.9 & 15.12 & 1.04 & 2.8 \\
60 & 1.76 & 17.9 & 3.88 & 4.2 & 22.51 & 1.36 & 3.9 \\
108 & 4.83 & 49.2 & 6.43 & 6.1 & 102.31 & 4.22 & 76.9 \\
159 & 6.19 & 63.1 & 7.28 & 5.4 & 148.44 & 4.50 & 89.8 \\
\hline \hline
\end{tabular}


This phenomenon can be observed regardless of the acceleration. It is also always found in exactly the same position and orientation. Therefore, it is likely triggered by a geometrical asymmetry in the nozzle, an extrinsic source. To test the possibility of an intrinsic source, one must achieve a purely RT-induced wave, which requires an optically transparent nozzle having well-controlled wetting conditions so that the nozzle jets entirely axisymmetrically. Also, a scaled-up system may be used, whereby small geometrical defects in the nozzle have a less pronounced effect on the fluid movement.

The constant contact-angle nozzle wall condition leads to the lowest acceleration threshold, which gives rise to the asymmetric sloshing mode $\kappa_{1,1}$. For experimental studies of RT instability the pinning boundary condition is often preferred, because it is easier to maintain than the $90^{\circ}$ contact-angle condition. In the inkjet experiments performed, however, the pinning is not controlled. For small actuations the contact line clearly remains pinned at the nozzle plate. However, in the experiments with stronger actuation where the meniscus retracts far into the nozzle, it is not possible to distinguish between a pinned meniscus and a moving meniscus as evident in Fig. 9. Finally, in the experiments performed here a sloshing mode is never observed. However, a growing protrusion is observed on the meniscus, which oscillates with a higher frequency around the meniscus. Finally this protrusion leads to the formation of a small high-velocity jet emerging from the center of the meniscus, as indicated in Fig. 9.

\section{CONCLUSIONS}

Asymmetric features in inkjet droplet formations can arise from intrinsic or extrinsic sources. Extrinsic sources include captured dust particles and imperfections in nozzle alignment and/or geometry. Intrinsic sources arise from the meniscus-formation process itself, features that can emerge in the absence of extrinsic geometric causes. After illustrating extrinsic asymmetry, the focus here has turned to the potential for asymmetric surface modes to arise intrinsically by the RT-instability mechanism.

The classical RT theory is rescaled to make general estimates for instabilities in inkjet nozzles. After first ascertaining that the meniscus moves on a slower timescale than the growth time of potentially relevant RT disturbances, Fig. 5 is used to further sharpen the potential of RT relevance. The figure can be used to answer for which accelerations the interface can become unstable and, if so, which wave numbers emerge. The selection of wave numbers by the local wetting and pinning condition and size of the nozzle allows only a subset of disturbance shapes. The growth rate for these shapes is estimated using the maximal growth rate $\sigma_{m}$. The gestation period, which is the duration the acceleration remains greater than the critical acceleration, determines how long the instability can grow. From these data the growth extent is estimated and the observability of a particular disturbance (relevance) is assessed.

The following interrogation sequence is proposed as a practitioner's guide to judging RT relevance. Questions 1-3 test for RT relevance by narrowing the class of disturbance, restricting by size and contacting. Questions 4-6 test feasibility using estimates obtainable via Fig. 10.

(1) Is a meniscus of some (any) extent unstable? That is, is RT potentially operative?

(2) If "yes," does the unstable meniscus fit into nozzle? In other words, is disturbance geometrically admissible or does confinement rule it out?

(3) If "yes," do contacting conditions accommodate the disturbance? More precisely, does wettability accommodate?

(4) If "yes," how fast does the admissible and accommodated disturbance grow?

(5) If fast growing, for how long can it grow before the mean meniscus decelerates sufficiently? That is, what is the gestation time period?

(6) If appreciable, does it grow enough to be seen? In other words, is the disturbance potentially observable?

As an illustration, these questions are applied to the reported experiments for a nozzle print head jetting water. The maximal acceleration and gestation period are obtained from the experiment and a maximal growth extent of 2285 is found (Table II), whereby one may conclude that RT waves can grow more than 800 -fold within the inkjet nozzles. This is facilitated by the acceleration during the in-stroke phase of the mean meniscus motion during the inkjet drop-on-demand actuation cycle. RT intrinsic asymmetry is relevant. In summary, using the six questions formulated and Fig. 5, the inkjet practitioner can determine which accelerations result in growth of waves on the meniscus and to what extent corresponding growth times can lead to observable disturbances.

\section{ACKNOWLEDGMENTS}

We thank Maaike Rump for assistance with the layout of the figures. P.S. thanks PoF for kind hospitality and the Dutch Research Centre of Fluid and Solid Mechanics (FSM) for supporting his visit to the University of Twente PoF. P.S. also thanks NSF CBET 1637960 for support during preparation of manuscript.

[1] J. R. Castrejon-Pita, W. Baxter, J. Morgan, S. Temple, G. D. Martin, and I. M. Hutchings, Future, opportunities and challenges of inkjet technologies, Atomization Spray 23, 541 (2013).

[2] K. Fukuda, T. Sekine, D. Kumaki, and S. Tokito, Profile control of inkjet printed silver electrodes and their 
application to organic transistors, ACS Appl. Mater. Interfaces 5, 3916 (2013).

[3] N. Chilton, in Inkjet Technology for Digital Fabrication (John Wiley \& Sons, Hoboken, New Jersey, United States, 2013), p. 183.

[4] H. C. Ki, S. H. Kim, D.-G. Kim, T.-U. Kim, S.-G. Kim, K.J. Hong, and S.-Y. So, in SPIE OPTO (SPIE, Bellingham, Washington, United States, 2013), p. 86221J.

[5] A. Teichler, R. Eckardt, S. Hoeppener, C. Friebe, J. Perelaer, A. Senes, M. Morana, C. J. Brabec, and U. S. Schubert, Combinatorial screening of polymer:fullerene blends for organic solar cells by inkjet printing, Adv. Energy Mater. 1, 105 (2011).

[6] M. Jung, J. Kim, J. Noh, N. Lim, C. Lim, G. Lee, J. Kim, H. Kang, K. Jung, A. D. Leonard, J. M. Tour, and G. Cho, Allprinted and roll-to-roll-printable 13.56-MHz-operated 1-bit RF tag on plastic foils, IEEE Trans. Electron Devices 57, 571 (2010).

[7] B. O'Neill, in Inkjet Technology for Digital Fabrication (John Wiley \& Sons, Hoboken, New Jersey, United States, 2013), p. 325.

[8] D. B. Wallace, in Inkjet Technology for Digital Fabrication (John Wiley \& Sons, Hoboken, New Jersey, United States, 2013), p. 141.

[9] C. A. Curcio, K. R. Sloan, R. E. Kalina, and A. E. Hendrickson, Human photoreceptor topography, J. Comput. Neurol. 292, 497 (1990).

[10] M. Vaezi, H. Seitz, and S. Yang, A review on 3D microadditive manufacturing technologies, J. Appl. Mech. 67, 1721 (2013).

[11] M. Caironi, E. Gili, and H. Sirringhaus, in Organic Electronics II (John Wiley \& Sons, Hoboken, New Jersey, United States, 2012), p. 281.

[12] Y. Wang, J. Bokor, and A. Lee, in Microlithography 2004, edited by R. Scott Mackay (SPIE, Bellingham, Washington, United States, 2004), p. 628.

[13] C. Menzel, P. Hoisington, and A. Bibl, in NIP20 (Society for Imaging Science and Technology (IS\&T), Springfield, USA, 2004), p. 169.

[14] A. L. Brady, M. M. McDonald, S. N. Theriault, and B. Smith, in NIP21 (Society for Imaging Science and Technology (IS\&T), Springfield, USA, 2005), p. 264.

[15] S. Berger, R. Burr, and D. Tence, Method and apparatus for producing dot size modulated ink jet printing, U.S. Patent No. 5,689,291 (1997).

[16] A. U. Chen and O. A. Basaran, A new method for significantly reducing drop radius without reducing nozzle radius in drop-on-demand drop production, Phys. Fluids 14, L1 (2002).

[17] M. W. Lee, D. K. Kang, N. Y. Kim, H. Y. Kim, S. C. James, and S. S. Yoon, A study of ejection modes for pulsed-DC electrohydrodynamic inkjet printing, J. Aerosol Sci. 46, 1 (2012).

[18] I. M. Hutchings and G. D. Martin, in Inkjet Technology for Digital Fabrication (John Wiley \& Sons, Hoboken, New Jersey, United States, 2013), p. 1.

[19] K. Suganuma, Introduction to Printed Electronics (Springer, New York, 2014).

[20] N. Link, S. Lampert, R. Gurka, A. Liberzon, G. Hetsroni, and R. Semiat, Ink drop motion in wide-format printers: II. Airflow investigation, Chem. Eng. Process. 48, 84 (2009).
[21] W.-K. Hsiao, S. D. Hoath, G. D. Martin, and I. M. Hutchings, in NIP28 (Society for Imaging Science and Technology (IS\&T), Springfield, USA, 2012), p. 412.

[22] A. A. Khalate, X. Bombois, R. Babuška, H. Wijshoff, and R. Waarsing, Performance improvement of a dropon-demand inkjet printhead using an optimization-based feedforward control method, Control Eng. Prac. 19, 771 (2011).

[23] A. K. Mogalicherla, S. Lee, P. Pfeifer, and R. Dittmeyer, Drop-on-demand inkjet printing of alumina nanoparticles in rectangular microchannels, Microfluidics Nanofluidics 16, 655 (2013).

[24] L. T. Creagh and M. McDonald, Design and performance of inkjet print heads for non-graphic-arts applications, MRS Bull. 28, 807 (2011).

[25] N. Link and R. Semiat, Ink drop motion in wide-format printers: I. Drop flow from Drop-On-Demand (DOD) printing heads, Chem. Eng. Process. 48, 68 (2009).

[26] J. R. Castrejón-Pita, N. F. Morrison, O. G. Harlen, G. D. Martin, and I. M. Hutchings, Experiments and Lagrangian simulations on the formation of droplets in drop-on-demand mode, Phys. Rev. E 83, 036306 (2011).

[27] O. G. Harlen and J. R. Castrejón-Pita, Asymmetric detachment from angled nozzles plates in drop-on demand inkjet printing, J. Imaging Sci. Technol. 29, 277 (2013).

[28] M. M. Fyrillas and A. J. Szeri, Dissolution or growth of soluble spherical oscillating bubbles, J. Fluid Mech. 277, 381 (1994).

[29] T. Leighton, The Acoustic Bubble (Academic Press, Cambridge, USA, 1997).

[30] J. de Jong, G. de Bruin, H. Reinten, M. van den Berg, H. Wijshoff, M. Versluis, and D. Lohse, Air entrapment in piezo-driven inkjet printheads, J. Acoust. Soc. Am. 120, 1257 (2006).

[31] D. Lohse, Bubble puzzles: From fundamentals to applications, Phys. Rev. Fluids 3, 110504 (2018).

[32] D. H. Kwon and S. J. Lee, Axis-switching of nonaxisymmetric microdroplet ejected from inkjet with an elliptical nozzle, Phys. Fluids 23, 051703 (2011).

[33] J. R. Castrejón-Pita, G. D. Martin, and I. M. Hutchings, Experimental study of the influence of nozzle defects on drop-on-demand ink jets, J. Imaging Sci. Technol. 55, 040305 (2011).

[34] Raleigh, Investigation of the character of the equilibrium of an incompressible heavy fluid of variable density, Proc. R. Soc. A s1-14, 170 (1882).

[35] G. Taylor, The instability of liquid surfaces when accelerated in a direction perpendicular to their planes. I, Proc. R. Soc. A 201, 192 (1950).

[36] R. D. Richtmyer, Taylor instability in shock acceleration of compressible fluids, Commun. Pure Appl. Math. 13, 297 (1960).

[37] A. Royon-Lebeaud, E. J. Hopfinger, and A. Cartellier, Liquid sloshing and wave breaking in circular and squarebase cylindrical containers, J. Fluid Mech. 577, 467 (2007).

[38] J. F. Dijksman and A. Pierik, in Inkjet Technology for Digital Fabrication (John Wiley \& Sons, Hoboken, New Jersey, United States, 2013), p. 45.

[39] M. Versluis, High-speed imaging in fluids, Exp. Fluids 54, 1 (2013). 
[40] A. van der Bos, A. Zijlstra, E. Gelderblom, and M. Versluis, iLIF: Illumination by laser-induced fluorescence for single flash imaging on a nanosecond timescale, Exp. Fluids 51, 1283 (2011).

[41] T. B. Benjamin, F. J. Ursell, and G. I. Taylor, The stability of the plane free surface of a liquid in vertical periodic motion, Proc. Roy. Soc. Lond. Ser. A: Math. Phys. Sci. 225, 505 (1954).
[42] M. P. Brenner, D. Lohse, and T. F. Dupont, Bubble Shape Oscillations and the Onset of Sonoluminescence, Phys. Rev. Lett. 75, 954 (1995).

[43] K. Kumar, Linear theory of faraday instability in viscous liquids, Proc. Roy. Soc. Lond. Ser. A: Math. Phys. Eng. Sci. 452, 1113 (1996).

[44] M.-J. van der Meulen, Ph.D. thesis, University of Twente, 2015. 